DOI:

10.1038/nri2060

\title{
IECs keep the peace
}

How can a protective immune response occur in the gut, where the immune cells must remain hyporesponsive to commensal bacteria and food antigens? Now, a report published in Nature shows that a mediator derived from intestinal-epithelial cells (IECs) regulates immune homeostasis in the gut following exposure to a pathogen.

Previous data have shown that IECs might have a role in the regulation of innate immunity in vitro, but the role of these cells in vivo was not known. Using a mouse model of intestinal nematode infection with Trichuris muris, the authors examined the role of IECs in the regulation of T-cell-mediated inflammation and immunity.

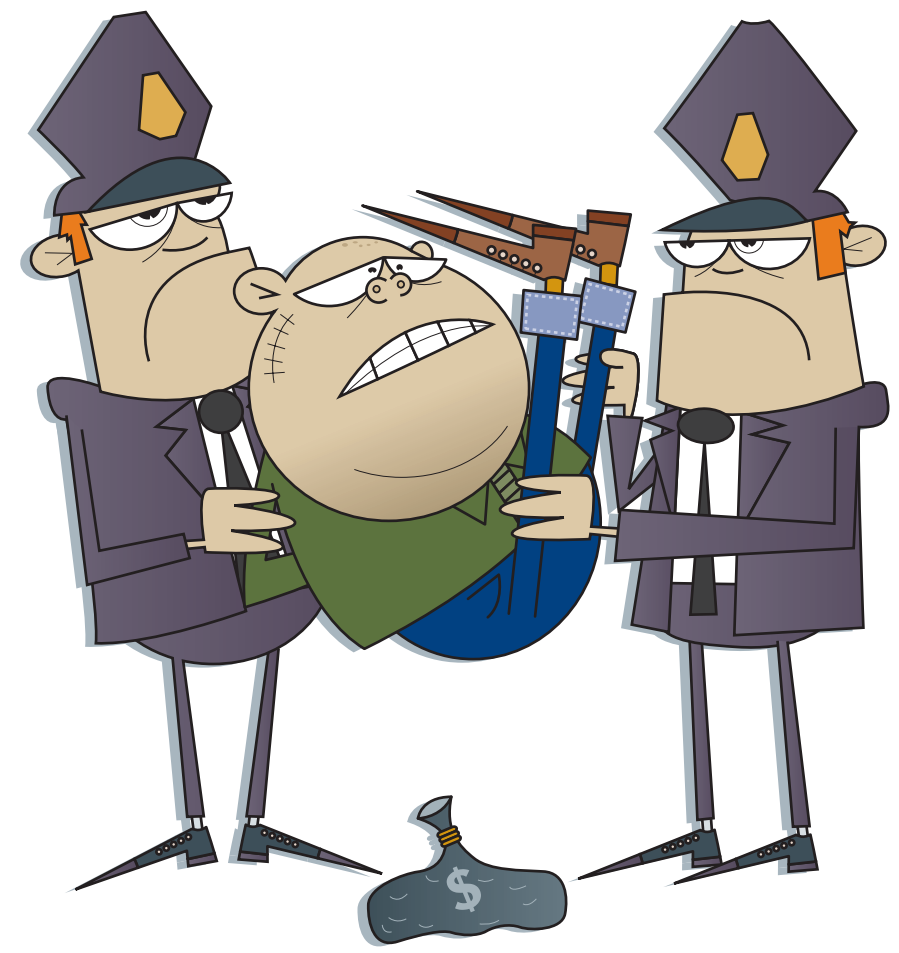

DNA-binding assays showed that in IECs, in vivo, T. muris induced the activation of nuclear factor- $\kappa \mathrm{B}$ $(N F-\kappa B)$ in an I $\kappa B$ kinase- $\beta$ (IKK $\beta)$ dependent manner. So, the authors generated mice in which IKK $\beta$ was specifically deleted in IECs (Ikkb $b^{\Delta \mathrm{IEC}}$ mice) to determine the influence of IKK $\beta$-dependent IEC-derived products on the immune response in the gut. $I k k b^{\triangle \mathrm{IEC}}$ mice infected with T. muris failed to mount a protective T helper $2\left(\mathrm{~T}_{\mathrm{H}} 2\right)$-cell response and clear the pathogen. In addition, the authors observed an increase in the production of the shared $\mathrm{p} 40$ subunit of interleukin-12 (IL-12) and IL-23 (known as IL-12/IL-23 p40) by dendritic cells (DCs), as well as interferon- $\gamma($ IFN $\gamma)$ and IL-17 by CD4 ${ }^{+} \mathrm{T}$ cells in $I k k b^{\triangle \mathrm{IEC}}$ mice compared with litter-mate control mice.

So, what IEC-derived factor(s) is absent in $I k k b^{\Delta \mathrm{IEC}}$ mice that could result in this dysregulated immune response? Gene analysis showed that the expression of IEC-derived thymic stromal lymphopoietin (TSLP) was significantly reduced in $I k k b^{\Delta \mathrm{IEC}}$ mice compared with wild-type mice. TSLP has previously been associated with the development of type 2 responses, but whether it acts by suppressing the production of pro-inflammatory cytokines or by directly inducing $\mathrm{T}_{\mathrm{H}}$ 2-cell-associated cytokines is not known. Conditioned supernatant from a mouse IEC line or recombinant TSLP inhibited the production of lipopolysaccharide-induced IL-12/IL-23 p40 production by DCs, indicating that the reduced expression of TSLP by IKK $\beta$-deficient IECs might be responsible for the enhanced production of pro-inflammatory cytokines in infected $I k k b^{\Delta \mathrm{IEC}}$ mice.

To confirm this hypothesis, $I k k b^{\Delta \mathrm{IEC}}$ mice infected with T. muris were treated with blocking antibodies specific for IL-12/IL-23 p40 and IFN $\gamma$. These mice were able to mount protective $\mathrm{T}_{\mathrm{H}} 2$-cell responses that resulted in clearance of the pathogen. The data indicate that in wildtype mice infected with T. muris, TSLP limits the production of pro-inflammatory cytokines by DCs and promotes the development of a protective $\mathrm{T}_{\mathrm{H}} 2$-cell response. Indeed, mice that lack the TSLP receptor were susceptible to infection with T. muris.

Therefore, the IKK $\beta$-dependent production of TSLP by IECs is crucial for regulating intestinal immune homeostasis after exposure to a gut pathogen, by directly influencing innate and adaptive immunity.

Olive Leavy

ORIGINAL RESEARCH PAPER Zaph, C. et al. Epithelial-cell-intrinsic IKK- $\beta$ expression regulates intestinal immune homeostasis. Nature 25 February 2007 (doi:10.1038/nature05590) 